\title{
Desarrollo post-embrionario, fecundidad y consumo de alimento de Dichroplus exilis (Orthoptera: Acrididae) bajo condiciones controladas
}

\author{
Christian Bardi ${ }^{1}$, Yanina Mariottini ${ }^{1}$, Maria L. De Wysiecki ${ }^{1}$ \& Carlos E. Lange ${ }^{1,2}$ \\ 1. Centro de Estudios Parasitológicos y de Vectores (CEPAVE) (CCT La Plata - CONICET - UNLP), Calle $2 \mathrm{~N}^{\circ} 584$, CP \\ 1900, La Plata, Buenos Aires, Argentina; bardi_c@yahoo.com.ar, ymariottini@cepave.edu.ar, mlw@cepave.edu.ar \\ 2. Comisión de Investigaciones Científicas (CIC) de la provincia de Buenos Aires; carlosl@cepave.edu.ar
}

$$
\text { Recibido 25-X-2010. C Corregido 20-II-2011. Aceptado 29-III-2011. }
$$

\begin{abstract}
Postembryonic development, fecundity and food consumption of Dichroplus exilis (Orthoptera: Acrididae) under controlled conditions. Dichroplus exilis is a widely distributed species in Southern South America. Although there have been reports of D. exilis as an agricultural pest, some recent observations suggest that the damage attributed to D. elongatus may actually have been caused by D. exilis. This study was conducted to determine the postembryonic life cycle stages, fertility and food consumption of this species under controlled conditions $\left(30^{\circ} \mathrm{C}, 14 \mathrm{~L}-10 \mathrm{D}, 40 \% \mathrm{RH}\right)$.Individuals employed belong to the laboratory-hatched first generation $(\mathrm{F} 1)$, from adults $(\mathrm{n}=64, \mathrm{\rho}=28, \hat{\delta}=36)$ collected in natural grasslands near Rafaela, Santa Fe province in NorthEastern Argentina. Three cohorts of 16, 17 and 20 individuals were monitored independently in acetate tubes on a daily basis, until death of the last insect. Average fecundity was 381.84, 38.54 eggs per female. Egg-pod incubation time was 14.4, 1.08 days and six nymphal instars were recorded. Nymphal development time was 41.38, 0.71 days $(I=8.73,0.20 ; \mathrm{II}=6.38,0.24 ; \mathrm{III}=5.64,0.33 ; \mathrm{IV}=7.15 ; 0.43 ; \mathrm{V}=9.76,0.54 ; \mathrm{IV}=7.85,0.95)$. The recorded food consumption was $9.89,1.08$ (mg/ind/day) for nymphs IV, 18.04, 0.73 ( $\mathrm{mg} / \mathrm{ind} /$ day) for nymphs V-IV, 16.76, $1.06(\mathrm{mg} / \mathrm{ind} /$ day) for pre-reproductive males, 28.09, $1.81(\mathrm{mg} / \mathrm{ind} /$ day) for pre-reproductive females, 7.71, 0.91 (mg/ind/day) for reproductive males and 13.06, 0.71 (mg/ind/day) for reproductive females, while the average adult food consumption, regardless of sex and reproductive status, was $16.41,4.32 \mathrm{mg} / \mathrm{day}$. Average food consumption of adult females was $17.47,1.15 \mathrm{mg}$, and was significantly higher than that of males $(10.83,0.91 \mathrm{mg})$. Data obtained in this study showed that $D$. exilis exhibits at least some of the biological attributes needed to configure an actual or potential agricultural pest, albeit not yet recognized as such. Field monitoring of grasshopper communities in areas where damage by D. exilis is suspected is envisaged in order to determine its possible status as a pest. Rev. Biol. Trop. 59 (4): 1579-1587. Epub 2011 December 01.
\end{abstract}

Key words: Agricultural pest, Argentina, consumption tests, Dichroplus elongatus, grasshopper, nymphal instars.

Dichroplus exilis (Giglio-Tos) es un melanoplino de amplia distribución geográfica en la región sur de América del Sur; se encuentra en Uruguay, Paraguay, Sur de Brasil (Rio Grande do Sul) y en el norte y gran parte del centro de la Argentina (Carbonell et al. 2006). D. exilis pertenece al "grupo elongatus" que incluye especies con fuerte similitud en la anatomía externa y los patrones de coloración (Ronderos et al. 1968). Es una especie poco estudiada, multivoltina, de régimen alimentario polífago y con diapausa embrionaria facultativa (Barrera \& Turk 1977, Turk \& Barrera 1979). Si bien hasta el presente no se han mencionado perjuicios económicos para el agro por parte de esta especie, es posible que daños a cultivos y pasturas debidos a su accionar hayan sido erróneamente atribuidos a Dichroplus elongatus (Giglio-Tos), especie simpátrica con la cual puede ser fácilmente confundida cuando la identificación se basa sólo en caracteres morfológicos externos (Ronderos et al. 1968, COPR 1982). 
La subfamilia Melanoplinae constituye el grupo más diverso de acridios en Argentina, con un total de 60 especies conocidas (Carbonell et al. 2006), muchas de las cuales suelen ser numéricamente dominantes en las comunidades de acridios del país, por ejemplo Cigliano et al. (2000), en el Oeste de la Región Pampeana registraron que la subfamilia Melanoplinae fue la más abundante, representando el $68.8 \%$ del total de individuos recolectados, mientras que De Wysiecki et al. (2004), determinaron que los melanoplinos representaron el $85 \%$ de los individuos en el Sudeste de la misma región. Dentro de esta familia, se encuentran algunas de las especies más perjudiciales (Dichroplus elongatus, Dichroplus maculipennis, Dichroplus pratensis, Dichroplus vitattus, Baeacris punctulatus, Ronderosia bergi y Scotussa lemniscata), para el agro argentino. (COPR 1982, Cigliano et al. 2002, Lange et al. 2005, Carbonell et al. 2006). No obstante, aún son limitadas las contribuciones que brindan información sobre aspectos biológicos de estas especies. Considerando esta situación, el objetivo del presente trabajo fue estudiar el ciclo de vida, la fecundidad y supervivencia de las hembras y el consumo de alimento de D. exilis bajo condiciones controladas.

\section{MATERIALES Y MÉTODOS}

Parámetros biológicos: Los individuos empleados en este estudio correspondieron a la primera generación (F1) obtenida en bioterio a partir de ejemplares adultos $(n=64$, $q=28, \hat{\delta}=36$ ), originalmente recolectados en pastizales naturales cercanos a la localidad de Rafaela, provincia de Santa Fe (31 ${ }^{\circ} 15^{\prime} 25.94$ » S - 61 $\left.{ }^{\circ} 29^{\prime} 14.48 » \mathrm{~W}\right)$, en el noreste de Argentina. Los acridios fueron criados en jaulas de aluminio de $(20 \times 20 \times 30 \mathrm{~cm})$ y alimentados con hojas frescas de lechuga, diferentes especies de gramíneas, repollo y salvado de trigo, y mantenidos bajo condiciones controladas $\left(30^{\circ} \mathrm{C}, 14 \mathrm{~L}: 10 \mathrm{O}, 40 \% \mathrm{HR}\right)$. La dieta y las condiciones ambientales fueron similares a las utilizadas en la mayoría de los centros de cría de acridios a nivel mundial (Henry 1985, Hinks \& Erlandson 1994).

Cada jaula fue provista de un sitio de oviposición para las hembras, que consistió en un recipiente cilíndrico de $8 \mathrm{~cm}$ de diámetro y $10 \mathrm{~cm}$ de profundidad, conteniendo como sustrato, partes iguales de arena y tierra tamizada y esterilizada. La termorregulación y la cópula fueron estimuladas con lámparas de $75 \mathrm{~W}$ suspendidas a unos $15 \mathrm{~cm}$ sobre las jaulas. Al carecer D. exilis de diapausa embrionaria obligatoria (Barrera \& Turk 1977, Turk \& Barrera 1979), las posturas fueron inmediatamente incubadas a $30^{\circ} \mathrm{C}$ hasta su nacimiento.

Para conocer el desarrollo post-embrionario se realizó el seguimiento diario de tres cohortes de 16, 17 y 20 individuos recién emergidos, hasta la muerte del último insecto, considerándose una cohorte a un conjunto de individuos de la misma edad. Cada cohorte fue mantenida de manera independiente en tubos transparentes de acetato con extremos de alambre tejido (Henry 1985) hasta alcanzar el estado adulto. Se registró la supervivencia diaria, la duración total del desarrollo postembrionario, el número y la duración de los estadios ninfales, la duración total del ciclo ninfal y la longevidad y supervivencia de las hembras adultas. Una vez en estado adulto se formaron 25 parejas para estimar la fecundidad. Cada pareja $(1 \hat{\jmath}, 1$ 우) fue colocada en una jaula de aluminio $(12 \times 12 \times 15 \mathrm{~cm})$ provista de un recipiente (sustrato) para la oviposición. Los sustratos fueron tamizados semanalmente para conocer el número promedio de posturas por hembra. Cincuenta posturas fueron abiertas bajo microscopio simple, con el fin de conocer el número de huevos por postura. Con estos datos se calculó el número de posturas por hembra a lo largo de toda su vida. El porcentaje de eclosión se estimó a partir de la incubación de siete posturas sin abrir y el posterior recuento de los huevos sin eclosionar presentes en las mismas.

Se construyeron las curvas de supervivencia (lx) y de fecundidad específica por edades $(\mathrm{mx})$ utilizando la porción hembra de la cohorte, y el intervalo de clases de edad sucesivas 
fue de una semana. A partir de estos datos se calculó la tasa neta de reproducción $\left(\mathrm{R}_{0}\right)$

$$
R_{0}=\sum_{x=1}^{n} l_{x} \cdot m_{x}=1
$$

Consumo: La estimación de consumo de alimento se realizó en ninfas de los últimos estadios (IV y V-VI), adultos pre-reproductivos y reproductivos. Dichos estadios y estados fueron seleccionados debido a que suelen ser los que mayores daños ocasionan (Putnam 1962, 1963, Sánchez \& De Wysiecki 1990). Las pruebas de consumo con adultos pre-reproductivos se realizaron con ejemplares de ambos sexos recién ingresados a imagos. Luego de los once días de pruebas con adultos pre-reproductivos, todos los individuos de ambos sexos fueron reunidos por un lapso de diez días, en el cual se observaron apareamientos (cópulas). Pasado este período, se separaron nuevamente por sexos para estimar el consumo de machos y hembras en estado reproductivo.

Para cada una de las pruebas de consumo se colocaron diez individuos en cada jaula de aluminio $(20 \times 20 \times 30 \mathrm{~cm})$, a los cuales se les ofreció alimento. Para ello, se prepararon raciones frescas de peso conocido de Bromus brevis Nees, especie nativa de Poaceae de valor forrajero (Ragonese 1985) y diez raciones testigo. El peso de cada ración se determinó con una balanza de precisión. Luego de 24h, el material remanente de cada ración fue retirado y secado en estufa $\left(60^{\circ} \mathrm{C}\right)$ hasta obtener peso constante. El peso seco promedio de las raciones testigo fue utilizado para calcular el factor de corrección, el cual fue aplicado al peso fresco inicial de cada una de las raciones ofrecidas para calcular el peso seco inicial. La diferencia de peso entre las raciones ofrecidas y el material remanente representó el material consumido. Se realizó un total de 191 pruebas (IV estadio $=33, \mathrm{~V}-\mathrm{VI}=45$, + pre-reproductivas $=16$, of reproductivas $=42$, of pre-reproductivos $=19$, $\delta$ reproductivos $=36$ ).

Los parámetros estimados fueron consumo promedio diario y total por individuo de cada estadio y, pérdida total promedio por individuo (consumo+material caído). El consumo de un individuo en la edad adulta se estimó promediando el consumo de los estados prereproductivo y reproductivo, multiplicado por la longevidad promedio. Se estimó la biomasa promedio de los individuos adultos de ambos sexos, expresada como peso seco, mediante la ponderación directa de los individuos, y se estimó la tasa de consumo relativo diaria (mg/ mg.individuo/día) para ambos sexos.

Los datos se analizaron mediante la prueba de Kruskal-Wallis seguido de Mann-Whitney. Todos los análisis se realizaron con XLSTAT 7.5.3. (Addinsoft 2005).

\section{RESULTADOS}

Parámetros biológicos: Se registró un total de seis estadios ninfales para ambos sexos (Fig. 1). La duración del estado ninfal fue de 41.38; Error Estándar 0.71; ámbito (34-51) días (Cuadro 1). Las primeras cópulas, manifestación externa de la madurez sexual en el macho (Richard \& Davies 1983), se observaron a partir del quinto día de haber alcanzado el estado adulto. La primera oviposición, indicio de la madurez sexual en la hembra (Michel \& Terán 2006), se observó a los once días de iniciado el estado adulto. El tiempo promedio de incubación fue de 14.4; 1.08; (9-19) días.

\section{CUADRO1}

Número y duración de los estadios ninfales de Dichroplus exilis bajo condiciones controladas

TABLE 1

Number and duration of the nymphal stages of Dichroplus exilis under controlled conditions

\begin{tabular}{cc} 
Estadio & Duración (días)* \\
I & $8.73 \pm 0.20$ \\
II & $6.38 \pm 0.24$ \\
III & $5.64 \pm 0.33$ \\
IV & $7.15 \pm 0.43$ \\
V & $9.76 \pm 0.54$ \\
VI & $7.85 \pm 0.95$ \\
Duración total del ciclo ninfal & $41.38 \pm 0.71$ \\
\hline
\end{tabular}

*= promedio \pm error estándar. 
A

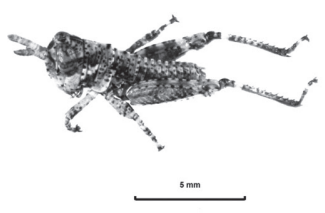

C

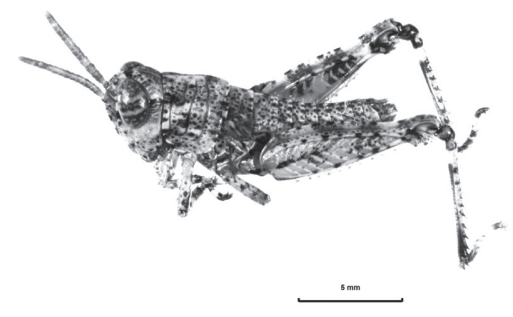

$E$

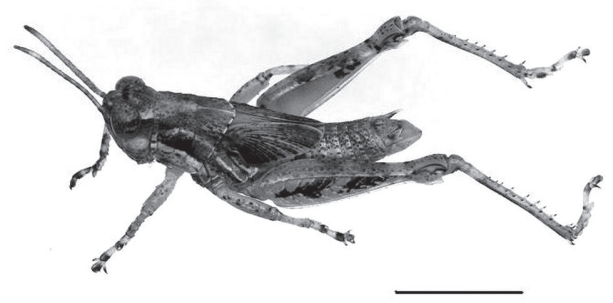

B

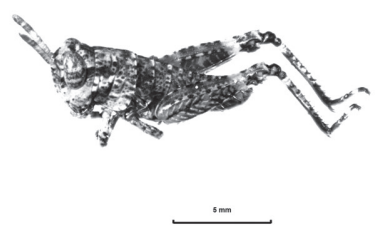

D

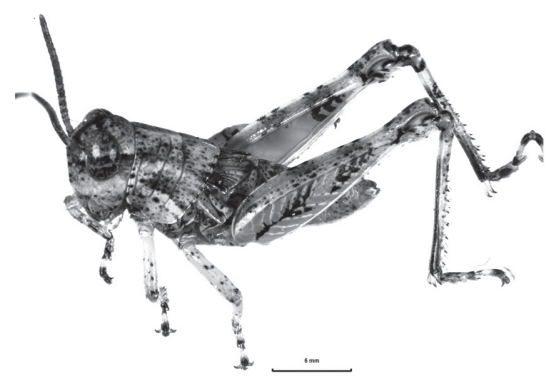

$\mathrm{F}$

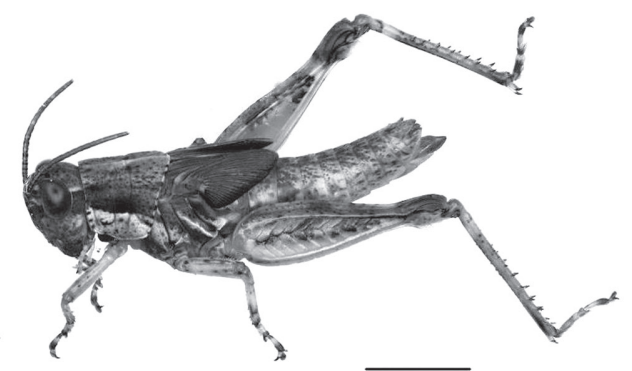

Fig. 1. Estadios ninfales de D. exilis. (A) Primero, (B) Segundo, (C) Tercero, (D) Cuarto, (E) Quinto, (F) Sexto. Fig. 1. Nymphal stages of D. exilis. (A) First, (B) Second, (C) Third, (D) Fourth, (E) Fifth, (F) Sixth.

El número promedio de posturas por hembra fue 19.96; 1.94; (0-37). El número promedio de huevos por postura fue $15.6 ; 1.03$; $(7-26)$. La fecundidad promedio (número de huevos/ hembra) fue 381.84, 38.54; (0-652) (Fig. 2). La tasa de oviposición fue de 2.36; 0.27 (huevos/ hembra/día). El porcentaje de eclosión fue de 94.33\%. La tasa neta de reproducción $\left(\mathrm{R}_{0}\right)$ fue de 176.78. La longevidad promedio de las hembras adultas fue de 158.64; 6.66; (48-194) días (Fig. 3). La duración promedio de las cohortes desde el nacimiento hasta la muerte fue de 201.56; 6.53 días.

Consumo: D. exilis no dejó caer comida al alimentarse, por lo que se consideró que la pérdida total fue igual al consumo. El contenido de agua de las raciones testigo (factor de corrección) fue de $80.03 ; 0.69 \%$. El consumo promedio fue de $9.89 ; 1.08$ (mg/ind./día) para las ninfas de IV estadio, 18.04; 0.73 (mg/ind./ día) para las ninfas de V-VI estadio, 16.76; 1.06 


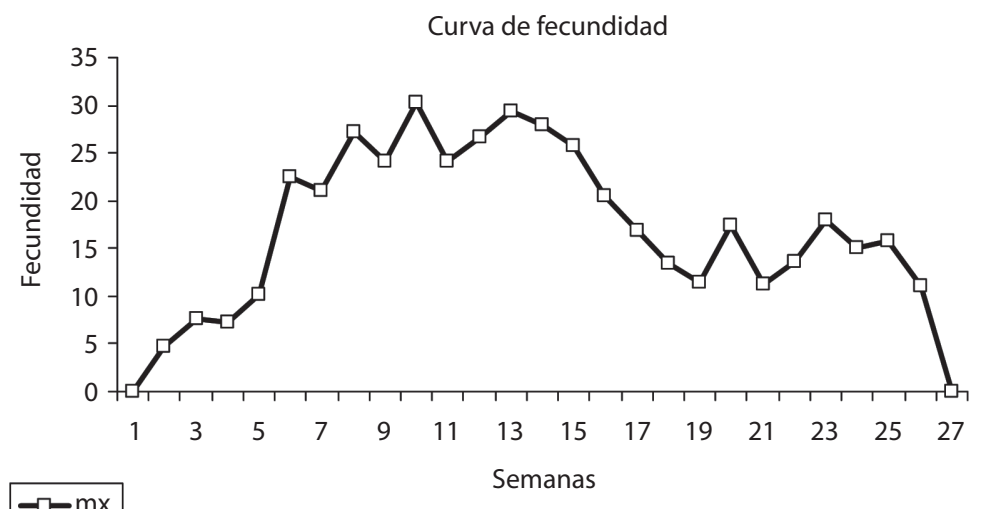

Fig. 2. Curva de fecundidad de D. exilis bajo condiciones controladas.

Fig. 2. Fertility curve of $D$. exilis under controlled conditions.

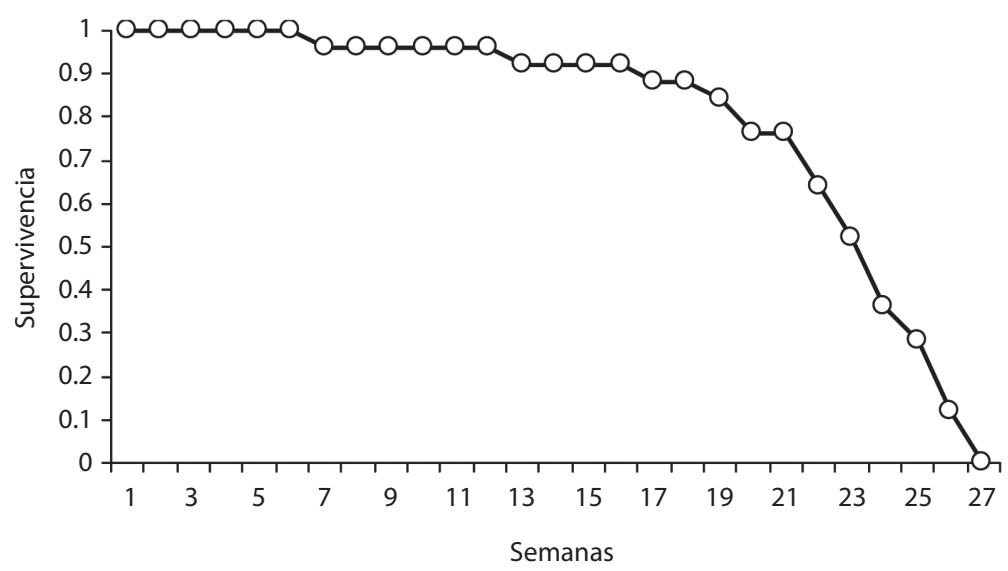

Fig. 3. Curvas de supervivencia de los individuos adultos hembra de D. exilis bajo condiciones controladas $\left(30^{\circ} \mathrm{C}, 14 \mathrm{~L}: 10 \mathrm{O}\right)$. Fig. 3. Survival curve of female adult D. exilis under controlled conditions $\left(30^{\circ} \mathrm{C}, 14 \mathrm{~L}: 10 \mathrm{O}\right)$.

(mg/ind./día) para los machos pre-reproductivos, 28.09; 1.81 (mg/ind./día) para las hembras pre-reproductivas, $7.71 ; 0.91(\mathrm{mg} /$ ind./día) para los machos reproductivos y 13.06; 0.71 (mg/ ind./día), para las hembras reproductivas. El consumo de los individuos en los distintos estadios de desarrollo fue significativamente diferente (Kruskal Wallis, $\mathrm{p}<0.0001$ ). Se observó un aumento en el consumo desde el IV estadio ninfal hasta los adultos pre-reproductivos, descendiendo en los adultos reproductivos (Fig. 4).
El consumo de las hembras pre-reproductivas ( ${ }_{+}$Pre) fue significativamente superior al consumo de los individuos en IV $(\mathrm{p}<0.001)$, V-VI $(\mathrm{p}<0.0001)$, ô Pre $(\mathrm{p}<0.0001)$, ㅇ Rep $(\mathrm{p}<0.0001)$ y $\widehat{\sigma} \operatorname{Rep}(\mathrm{p}<0.0001)$. El consumo de los machos pre-reproductivos ( $\widehat{\jmath}$ Pre) fue significativamente superior al consumo de los individuos en IV $(\mathrm{p}<0.001), \widehat{\jmath} \operatorname{Rep}(\mathrm{p}<0.0001)$, $q$ Rep ( $\mathrm{p}=0.003)$. El consumo de los individuos de V-VI fue significativamente superior al consumo de los individuos en IV $(\mathrm{p}<0.001)$, 


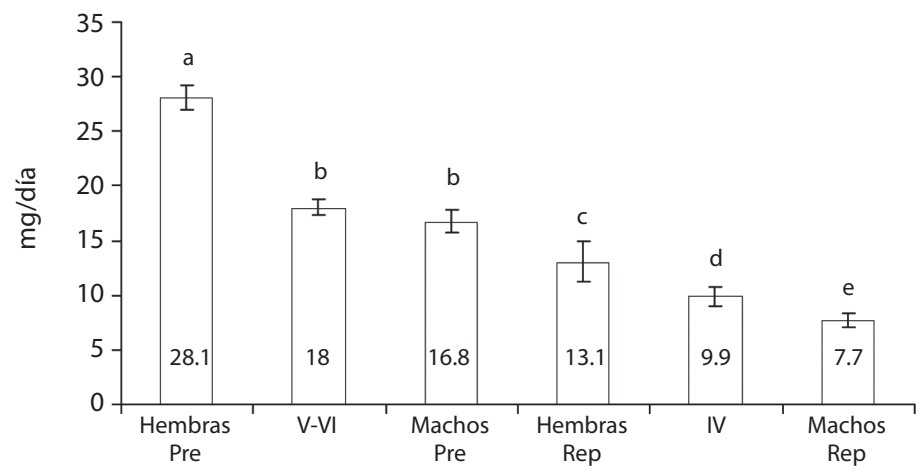

Fig. 4. Consumo promedio de los distintos estadios y estados D. exilis bajo condiciones controladas.

Fig. 4. Average consumption of the different stages and states of D. exilis under controlled conditions.

$\operatorname{Rep}(\mathrm{p}<0.0001)$ y $q \operatorname{Rep}(\mathrm{p}<0.0001)$. El consumo de las hembras reproductivas ( $ᄋ$ Rep) fue significativamente superior al consumo de los individuos en IV $(\mathrm{p}=0.043)$ y $\widehat{\sigma}$ Rep $(\mathrm{p}<0.0001)$. El consumo de los machos reproductivos ( $\hat{\sigma}$ Rep) fue significativamente inferior al de los individuos en IV ( $\mathrm{p}=0.026)$. No se registraron diferencias significativas entre el consumo de los individuos en V-VI y $\widehat{~ P r e ~}(\mathrm{p}=0.255)$. El consumo promedio de los adultos de $D$. exilis, sin diferenciar sexo y estado reproductivo, fue de $16.41 ; 4.32 \mathrm{mg} /$ día. El consumo promedio de una hembra en estado adulto fue de $17.47 ; 1.15 \mathrm{mg} /$ día, y resultó significativamente mayor que el de los machos, $10.83 ; 0.91 \mathrm{mg} /$ día (Kruskal-Wallis, $\mathrm{p}<0.001$ ). El consumo relativo en los adultos fue ligeramente mayor en los machos 0.263 (mg/ mg.individuo/día) que en las hembras 0.227 (mg/mg.individuo/día). La biomasa promedio de $D$. exilis fue de $90.74 ; 4.67 \mathrm{mg}$ para las hembras y $46.60 ; 1.32 \mathrm{mg}$ para los machos.

\section{DISCUSIÓN}

La Subfamilia Melanoplinae incluye el mayor número de especies de acridios en América, con aproximadamente 330 especies conocidas en América del Norte, unas 235 para América del Sur y alrededor de 80 en América Central (Otte 1995). Varias especies de melanoplinos, particularmente de los géneros Melanoplus y Dichroplus, constituyen serias plagas agrícolas en diferentes regiones del continente (COPR 1982, Pfadt 2002). Chapco et al. (2001) y Amédégnato et al. (2003) han postulado una estrecha relación filogenética entre Melanoplus y Dichroplus, mientras que Gangwere \& Ronderos (1978) y Vickery (1989) han sugerido cierta "equivalencia ecológica" entre ambos géneros. Por ello, resulta de interés comparar los resultados de este estudio con los obtenidos en otros emprendimientos realizados con especies afines bajo condiciones similares (temperatura, humedad, fotoperíodo, alimentación). Hasta el momento, los valores registrados en estudios acerca de los parámetros reproductivos en acridios del género Dichroplus diferían sustancialmente de los observados para Melanoplus. Por ejemplo, para Melanoplus sanguinipes en condiciones controladas, se han registrado 500-600 huevos por hembra durante todo el período de vida (Joern \& Gaines 1990), mientras que para Melanoplus bivittatus el promedio de huevos por hembra es de 450 (Pafdt 1994). En contraposición, los resultados obtenidos hasta el presente estudio para especies del género Dichroplus son muy inferiores. De Wysiecki et al. (1997) observaron para D. elongatus una fecundidad promedio 81.09; 14.02 huevos por hembra, Sánchez et al. (2001) registraron una fecundidad promedio de 
116.77; 32.60 para Dichroplus schulzi y Mariottini et al. (2008) y Mariottini (2009) registraron valores de $46.03 ; 6.84$ y de $86.06 ; 13.03$ para Beacris (Dichroplus) pseudopunctulatus y Dichroplus maculipennis, respectivamente. Dichroplus exilis, en cambio, presentó un promedio de 381.84, 38.54 huevos por hembra, con un máximo de 652huevos/hembra. Estos valores resultan más cercanos a los observados para Melanoplus que para otros Dichroplus.

Los acridios figuran entre los principales consumidores primarios en los sistemas de pastizal (Hewitt \& Onsager 1983, O’Neill et al. 2003), llegando a competir por el recurso forrajero con el ganado cuando alcanzan altas densidades (De Wysiecki \& Sánchez 1992, Fielding \& Brusven 1995, O’Neill et al. 2003). Si comparamos los resultados de este estudio con aquellos obtenidos para $D$. elongatus y D. maculipennis (dos de las especies más perjudiciales en Argentina) en condiciones similares (Cuadro 2), vemos que la pérdida total (material consumido+material caído) es menor para $D$. exilis. Sin embargo, cuando tomamos la tasa de consumo relativa, el consumo se asemeja y hasta supera a las tasas de consumo de las otras especies comparadas.

Analizando los resultados en el marco teórico aportado por Hewitt \& Onsager (1983), quienes sostienen que la pérdida de forraje producido por acridios está básicamente gobernada por la densidad de la población de la especies involucradas, las tasas de consumo y la longevidad de los adultos, se puede concluir que $D$. exilis es una potencial especie plaga que debe ser tomada en cuenta, ya que se trata de individuos longevos, con una elevada fecundidad y un consumo semejante al de $D$. elongatus. Sumado a esto, debe tenerse en cuenta a la hora de analizar el posible estatus

CUADRO 2

Consumo promedio de tres especies de Dichroplus bajo condiciones controladas

TABLE 2

Average consumption of three species of Dichroplus under controlled conditions

\begin{tabular}{|c|c|c|c|c|}
\hline Especie & Estadio & $\begin{array}{c}\text { Pérdida Total } \\
(\text { consumo }+ \\
\text { material caído)* }\end{array}$ & $\begin{array}{l}\text { Pérdida total } \\
\text { del estadio }\end{array}$ & $\begin{array}{l}\text { Tasa de consumo } \\
\text { (mg/mg-ind/día) }\end{array}$ \\
\hline \multirow{5}{*}{$\begin{array}{l}\text { Dichroplus maculipennis } \\
\text { (Mariottini 2009) }\end{array}$} & V & $24.5 \pm 1.23$ & 145.5 & 0.47 \\
\hline & $\lesssim$ (prereproductivos) & $33.5 \pm 0.9$ & 1760.4 & 0.17 \\
\hline & $\widehat{\delta}$ (reproductivos) & $12.8 \pm 0.45$ & & 0.06 \\
\hline & q (prereproductivos) & $49.1 \pm 0.7$ & 2360.8 & 0.15 \\
\hline & q (reproductivos) & $18.9 \pm 0.8$ & & 0.06 \\
\hline \multirow{6}{*}{$\begin{array}{l}\text { Dichroplus elongatus } \\
\text { (Mariottini 2009) }\end{array}$} & IV & $13 \pm 0.46$ & 104.2 & 0.47 \\
\hline & V & $21.3 \pm 0.48$ & 149.4 & 0.35 \\
\hline & $\widehat{\jmath}$ (prereproductivos) & $20.4 \pm 0.37$ & 1002.5 & 0.28 \\
\hline & $\widehat{o}$ (reproductivos) & $12.9 \pm 0.31$ & & 0.18 \\
\hline & q (prereproductivos) & $30.6 \pm 0.56$ & 832.2 & 0.23 \\
\hline & q (reproductivos) & $19.1 \pm 0.05$ & & 0.15 \\
\hline \multirow[t]{6}{*}{ Dichroplus exilis } & IV & $9.89 \pm 1.08$ & 63.7 & - \\
\hline & V - VI & $18.04 \pm 0.73$ & 317.7 & - \\
\hline & $\delta$ (prereproductivos) & $16.76 \pm 1.06$ & - & 0.36 \\
\hline & $\lesssim$ (reproductivos) & $7.71 \pm 0.91$ & & 0.16 \\
\hline & q (prereproductivos) & $28.09 \pm 1.81$ & 3272.2 & 0.31 \\
\hline & + (reproductivos) & $13.06 \pm 0.71$ & & 0.14 \\
\hline
\end{tabular}

*=promedio \pm error estándar. 
de D. exilis como plaga, que la misma carece de diapausa embrionaria obligatoria, posibilitándole tener más de una generación anual, lo que ciertamente puede generar un incremento en el daño ocasionado.

Será necesario realizar prospecciones en las zonas donde se presume que $D$. exilis es la especie realmente causante de daños y no $D$. elongatus, tales como la provincia de Santa $\mathrm{Fe}$ y el noroeste de la región Pampeana (noroeste de Buenos Aires, Norte de La Pampa, Sur de Córdoba), para dilucidar su eventual estatus como plaga.

Adicionalmente, dado que $D$. exilis carece de diapausa embrionaria obligatoria y tiene en laboratorio alta longevidad y fecundidad puede ser considerada como una especie apta para el desarrollo y mantenimiento de colonias de cría, las cuales son de utilidad para la realización de estudios experimentales, en particular con agentes de control biológico (Lange 2002, 2003).

\section{AGRADECIMIENTOS}

Esta investigación fue financiada en parte por el "Fondo para la Investigación Científica y Tecnológica (FONCYT, proyecto PICT 2006/679)" y "Comisión de Investigaciones Científicas (CIC) de la provincia de Buenos Aires".

\section{RESUMEN}

La Subfamilia Melanoplinae tiene una relevancia central dentro de la acridiofauna Argentina. Varias especies suelen ser numéricamente dominantes en las comunidades de acridios del país y algunas constituyen serias plagas. El objetivo de este trabajo fue conocer el ciclo de vida postembrionario, la fecundidad y supervivencia de las hembras y el consumo de alimento de Dichruplus exilis bajo condiciones controladas $\left(30^{\circ} \mathrm{C} ; 40 \% \mathrm{HR}\right.$ y $\left.14 \mathrm{~L}-10 \mathrm{O}\right)$. Para ello se realizó el seguimiento de tres cohortes de 16, 17 y

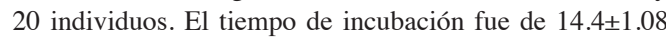
días. La duración del desarrollo ninfal fue 41.38; 0.71 días (I 8.73; 0.20, II 6.38; 0.24, III 5.64; 0.33, IV 7.15; 0.43, V 9.76; 0.54; IV 7.85; 0.95); la fecundidad promedio fue de 381.84, 38.54huevos/hembra. El consumo registrado fue de $9.89 ; 1.08$ (mg/ind./día) para las ninfas de IV, 18.04; 0.73 (mg/ind./día) para las de V y IV, mientras que el consumo promedio de los adultos, sin diferenciar sexo y estado reproductivo, fue de $16.41 ; 4.32 \mathrm{mg}$ /día. Estos parámetros, al ser comparados con los de otras especies afines, indican que $D$. exilis exhibe atributos biológicos que condicen con los de otros melanoplinos considerados plaga.

Palabras clave: Acridio, Argentina, Dichroplus elongatus, estadio ninfal, plaga agrícola, pruebas de consumo.

\section{REFERENCIAS}

Addinsoft. 2005. XLSTAT Pro version 7.5.3 (disponible en línea: http://www.xlstat.com/en/home).

Amédégnato, C., W. Chapco \& G. Litzenberger. 2003. Out of South America? Additional evidence for a southern origin of melanopline grasshoppers. Mol. Phylogenet. Evol. 29: 115-119.

Barrera, M. \& S.Z. Turk. 1977. Acridios del NOA II: Contribución al conocimiento de huevos, desove y hábitos de postura de algunas especies de tucuras (Orthoptera: Acrididae) de la provincia de Tucumán. Acta Zool. Lilloana 32: 167-188.

Carbonell, C.S., M.M. Cigliano \& C.E. Lange. 2006. Acridomorph (Orthoptera) species of Argentina and Uruguay. CD-ROM. Publications on Orthopteran Diversity, The Orthopterists Society at the "Museo de La Plata", Argentina.

Chapco, W., G. Litzenberger \& W.R. Kuperus. 2001. A molecular biogeographic analysis of the relationship between North American melanopline grasshoppers and their Eurasian and South American relatives. Mol. Phylogenet. Evol. 18: 460-466

Cigliano, M.M., M.L. De WysieckI \& C.E. Lange. 2000. Grasshopper (Orthoptera, Acrididae) species diversity in the Pampas, Argentina. Divers. Distrib. 6: 81-91.

Cigliano, M.M., S. Torrusio \& M.L. De Wysiecki. 2002. Grasshopper (Orthoptera: Acrididoidea) community composition and temporal variation in The Pampas, Argentina. J. Orthop. Res. 11: 215-221.

Centre for Overseas Pest Research (COPR). 1982. The locust and grasshopper agricultural manual, COPR, London.

De Wysiecki, M.L. \& N. Sánchez. 1992. Dieta y remoción de forraje de Dichroplus pratensis (Orthoptera, Acrididae) en un pastizal de la provincia de La Pampa, Argentina. Ecol. Austral. 2: 19-27.

De Wysiecki, M.L., M.M. Cigliano \& C.E. Lange. 1997. Fecundidad y longevidad de adultos de Dichroplus 
elongatus (Orthoptera: Acrididae) bajo condiciones controladas. Rev. Soc. Entomol. Argent. 56: 101-104.

De Wysiecki, M.L., S. Torrusio \& M.M. Cigliano. 2004. Caracterización de las comunidades de acridios del partido de Benito Juárez, sudeste de la provincia de Bs. As, Argentina. Rev. Soc. Entomol. Argent. 63: 87-96.

Fielding, D.J. \& M.A. Brusven. 1995. Ecological correlates between rangeland grasshopper (Orthoptera: Acrididae) and plant communities of southern Idaho. Environ. Entomol. 24: 1432-1441.

Gangwere, S.K. \& R.A. Ronderos. 1978. The current status of New World acridology. Interciencia 3: 16-19.

Henry, J.E. 1985. Melanoplus spp., p. 451-464. In P. Singh \& R.F. Moore (eds.). Handbook of insect rearing, Vol. 1. Elsevier, Amsterdam.

Hewitt, G.B. \& R.J. Onsager. 1983. Control of grasshopper on rangeland in the United States, a perspective. J. Range Manage. 3: 202-207.

Hinks, C.F. \& M.A. Erlandson. 1994. Rearing grasshoppers and locust: review, rationale and update. J. Orthop. Res. 3: 1-10.

Joern, A. \& S.B. Gaines. 1990 Populations dynamics and regulation in grasshoppers, p. 415-482. In R.F. Chapman \& A. Joern (eds.). Biology of grasshoppers. John Wiley and Sons, New York, EEUU.

Lange, C.E. 2002. La amebiasis debilitativa de los ortópteros y su potencial para el control biológico de acridios (Orthoptera: Acridoidea) en la Argentina. Rev. Invest. Agropecu., Ser. 2. 31: 25-38.

Lange, C.E. 2003. Niveles de esporulación experimentales y naturales del agente de biocontrol Nosema locustae (Protozoa. Microspora) en especies de tucuras y langostas (Orthoptera: Acridoidea) de la Argentina. Rev. Soc. Entomol. Argent. 62: 15-22.

Lange, C.E., M.M. Cigliano \& M.L. De Wysiecki. 2005. Los acridoideos de importancia económica en la Argentina, p. 93-135. In L. Barrientos Lozano \& P. Almaguer (eds.). Manejo integrado de la langosta centroamericana y acridoideos plaga en América Latina. Instituto Tecnológico de Ciudad Victoria, Tamaulipas, México.

Michel, A.A. \& H.R. Terán. 2006. Cría en cautiverio de Baeacris punctulatus (Orthoptera,Acrididae, Melanoplinae). Acta Zool. Lilloana 50: 131-134.

Mariottini, Y., M.L. De Wysiecki \& C.E. Lange. 2008. Fecundidad y desarrollo postembrionario de Baeacris pseudopunctulatus Ronderos (Orthoptera: Acrididae: Melanoplinae) bajo condiciones controladas. Rev. Soc. Entomol. Argent. 67: 135-138.
Mariottini, Y. 2009. Biología y ecología de Acridios (Orthoptera: Acridoidea). Tesis Doctorado, Universidad Nacional de La Plata, La Plata, Buenos Aires, Argentina.

O’Neill, K.M., B.E. Olson, M.G. Rolston, R. Wallander, D.P. Larson \& C.E. Seibert. 2003. Effects of livestock grazing on rangeland grasshopper (Orthoptera:Acrididae) abundance. Agr. Ecosyst. Environ. 97: 51-64.

Otte, D. 1995. Orthoptera species File 4. Grasshoppers [Acridomorpha] C. Orthopterist's Society and The Academy of Natural Sciences of Philadelphia, Philadelphia, EEUU.

Pfadt, R.E. 1994. Migratory grasshopper Melanoplus sanguinipes (Fabricius). Wyoming Agricultural Experiment Station Bulletin 912, Species Fact Sheet. (disponible en línea: www.sdvc.uwyo.edu/grasshopper/mesa.htm).

Pfadt, R.E. 2002. Field guide to common western grasshoppers. University of Wyoming, Laramie, EEUU.

Putnam, L.G. 1962. The damage potential of some grasshoppers on the Native Grassland of British Columbia. Can. J. Plant. Sci. 42: 596-601.

Putnam, L.G. 1963. The progress of nymphal development in pest grasshoppers (Acrididae) of western Canada. Can. Entomol. 95: 1210-1216.

Ragonese, E.A. 1985. Forrajeras nativas. Reunión Técnica en Honor al Ing. Hermán Serrano. INTA EEA Pergamino.

Ronderos, R.A., C.S. Carbonell \& A. Mesa. 1968. Revisión de las especies del género Dichroplus Stal, del grupo elongatus (Orthoptera, Acrididae, Catantopinae). Rev. Mus. La Plata, Secc. Zool. 10: 271-325.

Sánchez, N.E. \& M.L. De Wysiecki. 1990. Quantitative evaluation of feeding activity of the grashopper Dichroplus pratensis (Orthoptera: Acrididae) in a natural grassland of La Pampa, Argentina. Environ. Entomol. 19: 1392-1395.

Sánchez, N.E., P. Pessacq, M. Theiller \& G. Almandoz. 2001. Aspectos biológicos y demografía de Dichroplus schulzi Bruner (Orthoptera: Acrididae), en laboratorio. Rev. Soc. Entomol. Argent. 60: 73-78.

Turk, S.Z. \& M. Barrera. 1979. Acridios del NOA. III. Estudio bio-ecológico sobre siete especies del género Dichroplus Stal (Orthoptera, Acrididae). Acta Zool. Lilloana 35: 785-805.

Vickery, V.R. 1989. The biogeography of Canadian Grylloptera and Orthoptera. Can. Entomol. 121: 389-424. 
